\title{
Cosmic vector for dark energy
}

\author{
Jose Beltrán Jiménez and Antonio L. Maroto \\ Departamento de Física Teórica, Universidad Complutense de Madrid, 28040 Madrid, Spain
}

(Received 15 January 2008; published 8 September 2008)

\begin{abstract}
In this work we show that the presence of a vector field on cosmological scales could explain the present phase of accelerated expansion of the Universe. The proposed theory contains no dimensional parameters nor potential terms and does not require unnatural initial conditions in the early universe, thus avoiding the so-called cosmic coincidence problem. In addition, it fits the data from high-redshift supernovae with excellent precision, making definite predictions for cosmological parameters. Upcoming observations will be able to clearly discriminate this model from standard cosmology with cosmological constant.
\end{abstract}

DOI: 10.1103/PhysRevD.78.063005

PACS numbers: $95.36 .+\mathrm{x}, 98.80 . \mathrm{Cq}$

Recent cosmological observations [1-4] indicate that the Universe is undergoing a phase of accelerated expansion. The fact that the present rate of expansion is accelerating rather than decelerating poses one of the most important problems of modern cosmology. Indeed, in standard cosmology our Universe starts expanding after the big bang, but the attractive nature of gravity for ordinary matter and radiation necessarily slows down the expansion rate. In order to have acceleration, Einstein's equations require the Universe to be dominated by some sort of nonordinary energy (usually called dark energy) with the particular property of having negative pressure.

Although its nature is unknown, a simple phenomenological description in which dark energy is understood as a cosmological constant, i.e. a perfect fluid with equation of state, $p_{\Lambda}=-\rho_{\Lambda}$, where $\rho_{\Lambda}$ and $p_{\Lambda}$ are the energy density and pressure, respectively, seems to fit observations with very good precision ( $\Lambda \mathrm{CDM}$ model). Thus, $\Lambda \mathrm{CDM}$ suggests that around $70 \%$ of the energy density of the Universe today would be in the form of dark energy, whereas the remaining $30 \%$ would be nonrelativistic matter (the contribution from radiation and curvature being negligible) [3].

However the fact that today matter and dark energy have comparable contributions to the energy density [both around $\left(10^{-3} \mathrm{eV}\right)^{4}$ in $\hbar=c=1$ units] turns out to be difficult to understand if dark energy is a true cosmological constant. Indeed, the energy density of a cosmological constant remains constant throughout the history of the Universe, whereas those of the rest of components (matter or radiation) grow as we go back in time. Then the question arises as to whether it is a coincidence (or not) that they have comparable values today when they have differed by many orders of magnitude in the past. In addition, the cosmological constant exhibits another related problem. Its scale (around $10^{-3} \mathrm{eV}$ ) is more than 30 orders of magnitude smaller than the scale of the other dimensional constant appearing in the gravitational equations, $G=$ $M_{P}^{-2}$ with $M_{P} \sim 10^{19} \mathrm{GeV}$, and it is also difficult to explain from particle or other known physics.
In order to avoid these problems several models have been proposed in which dark energy is a dynamical component rather than a cosmological constant. Such models are usually based on cosmological scalar fields or modifications of Einstein's gravity [5-8]. However, in order to have acceleration at the right time, they typically introduce unnatural dimensional scales, resulting once again in finetuning or coincidence problems.

In this paper we consider a completely different type of dark energy model which is not based on scalar fields, but in the dynamics of a vector field. Unlike previous works $[9,10]$, it is shown that vector fields can give rise to periods of acceleration even in the absence of potential terms (see [11] for a general analysis). The existence of such solutions does not rely on the introduction of complicated functions of the fields and its derivatives, but can be obtained with the simplest kinetic terms, including two fields and two derivatives, so that the model does not contain dimensional parameters. Furthermore, we show that the required initial conditions for the vector fields are natural.

Let us start by writing the action of our vector-tensor theory of gravity containing only two fields and two derivatives and without potential terms [12]:

$$
\begin{aligned}
S= & \int d^{4} x \sqrt{-g}\left(-\frac{R}{16 \pi G}-\frac{1}{2} \nabla_{\mu} A_{\nu} \nabla^{\mu} A^{\nu}\right. \\
& \left.+\frac{1}{2} R_{\mu \nu} A^{\mu} A^{\nu}\right) .
\end{aligned}
$$

Notice that the theory contains no free parameters, the only dimensional scale being Newton's constant. The numerical factor in front of the vector kinetic terms can be fixed by the field normalization. Also notice that $R_{\mu \nu} A^{\mu} A^{\nu}$ can be written as a combination of derivative terms as $\nabla_{\mu} A^{\mu} \nabla_{\nu} A^{\nu}-\nabla_{\mu} A^{\nu} \nabla_{\nu} A^{\mu}$

The classical equations of motion derived from the action in (1) are the Einstein's and vector field equations:

$$
R_{\mu \nu}-\frac{1}{2} R g_{\mu \nu}=8 \pi G\left(T_{\mu \nu}+T_{\mu \nu}^{A}\right)
$$




$$
\square A_{\mu}+R_{\mu \nu} A^{\nu}=0,
$$

where $T_{\mu \nu}$ is the conserved energy-momentum tensor for matter and radiation and $T_{\mu \nu}^{A}$ is the energy-momentum tensor coming from the vector field. In this work we shall solve these equations for the simplest isotropic and homogeneous flat cosmologies. Thus, we assume that the spatial components of the vector field vanish, so that $A_{\mu}=$ $\left(A_{0}(t), 0,0,0\right)$ and, therefore, the space-time geometry will be given by the flat Robertson-Walker metric:

$$
d s^{2}=d t^{2}-a^{2}(t) \delta_{i j} d x^{i} d x^{j}
$$

For this metric (3) reads

$$
\ddot{A}_{0}+3 H \dot{A}_{0}-3\left[2 H^{2}+\dot{H}\right] A_{0}=0,
$$

where $H=\dot{a} / a$ is the Hubble parameter.

Assuming that the Universe has gone through radiation and matter phases in which the contribution from dark energy was negligible, we can easily solve these equations in those periods just taking $H=p / t$, with $p=1 / 2$ for radiation and $p=2 / 3$ for matter eras, respectively, which is equivalent to assuming that $a \propto t^{p}$. In that case, the above equation has a growing and a decaying solution:

$$
A_{0}(t)=A_{0}^{+} t^{\alpha_{+}}+A_{0}^{-} t^{\alpha_{-}},
$$

with $A_{0}^{ \pm}$constants of integration and $\alpha_{ \pm}=-(1 \pm 1) / 4$ in the radiation era, and $\alpha_{ \pm}=(-3 \pm \sqrt{33}) / 6$ in the matter era.

On the other hand, the (00) component of Einstein's equations reads

$$
H^{2}=\frac{8 \pi G}{3}\left[\sum_{\alpha} \rho_{\alpha}+\rho_{A}\right]
$$

with $\alpha=M, R$ and

$$
\rho_{A}=\frac{3}{2} H^{2} A_{0}^{2}+3 H A_{0} \dot{A}_{0}-\frac{1}{2} \dot{A}_{0}^{2} .
$$

Using the growing mode solution in (6), we obtain

$$
\rho_{A}=\rho_{A 0} a^{\kappa},
$$

with $\kappa=-4$ in the radiation era and $\kappa=(\sqrt{33}-9) / 2 \simeq$ -1.63 in the matter era. Thus the energy density of the vector field starts scaling as radiation at early times, so that $\rho_{A} / \rho_{R}=$ const. However when the Universe enters its matter era, $\rho_{A}$ starts growing relative to $\rho_{M}$ eventually overcoming it at some point, in which the dark energy vector field would become the dominant component. From that point on, we cannot obtain analytic solutions to the field equations. In Fig. 1 we show the numerical solution to the exact equations, which confirms our analytical estimates in the radiation and matter eras. Notice that since $A_{0}$ is essentially constant during the radiation era, solutions do not depend on the precise initial time at which we specify it. Thus, once the present value of the Hubble parameter $H_{0}$ and the constant $A_{0}$ during radiation

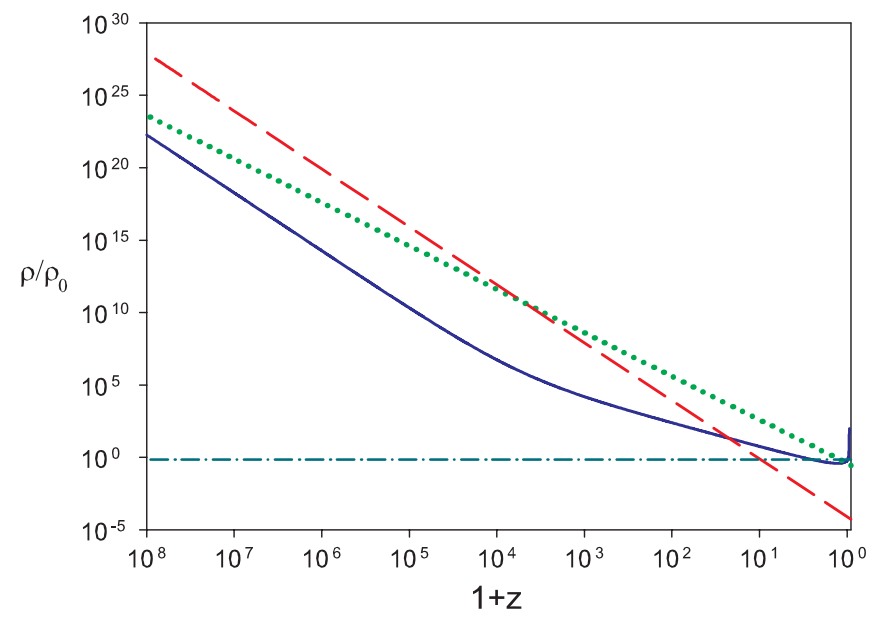

FIG. 1 (color online). Evolution of energy densities for the best-fit model. Dashed (red) line for radiation, dotted (green) line for matter, and solid (blue) line for vector dark energy. We show also for comparison the cosmological constant density in a dashed-dotted line. We see the rapid growth of the vector dark energy contribution at late times approaching the final singularity.

(which fixes the total matter density $\Omega_{M}$ ) are specified, the model is completely determined. In other words, this model contains the same number of parameters as $\Lambda \mathrm{CDM}$, i.e. the minimum number of parameters of a cosmological model with dark energy. As seen from Fig. 1 the evolution of the Universe ends at a finite time $t_{\text {end }}$ with a singularity in which $a \rightarrow a_{\text {end }}$ with $a_{\text {end }}$ finite, $\rho_{\mathrm{DE}} \rightarrow \infty, p_{\mathrm{DE}} \rightarrow-\infty$, and $A_{0}\left(t_{\mathrm{end}}\right)=M_{P} /(4 \sqrt{\pi})$. This corresponds to a type III singularity according to the classification in [13].

We can also calculate the effective equation of state for dark energy as

$$
w_{\mathrm{DE}}=\frac{p_{A}}{\rho_{A}}=\frac{-3\left(\frac{5}{2} H^{2}+\frac{4}{3} \dot{H}\right) A_{0}^{2}+H A_{0} \dot{A}_{0}-\frac{3}{2} \dot{A}_{0}^{2}}{\frac{3}{2} H^{2} A_{0}^{2}+3 H A_{0} \dot{A}_{0}-\frac{1}{2} \dot{A}_{0}^{2}} .
$$

Again, using the approximate solutions in (6), we obtain

$$
w_{\mathrm{DE}}=\left\{\begin{array}{ll}
\frac{1}{3} & \text { radiation era } \\
\frac{3 \sqrt{33}-13}{\sqrt{33}-15} \simeq-0.457 & \text { matter era }
\end{array} .\right.
$$

After dark energy starts dominating, the equation of state abruptly falls towards $w_{\mathrm{DE}} \rightarrow-\infty$ as the Universe approaches $t_{\text {end }}$. As shown in Fig. 2 the equation of state can cross the so-called phantom divide, so that we can have $w_{\mathrm{DE}}(z=0)<-1$. In Fig. 3, we show the evolution of the $A_{0}$ component.

In order to confront the predictions of the model with observations of high-redshift type Ia supernovae, we have calculated the distance modulus as a function of redshift. Comparing $\mu_{\mathrm{th}}(z)$ with its observational value in a given data set will enable us to carry out a $\chi^{2}$ statistical analysis. For this purpose, we have considered two sets of super- 


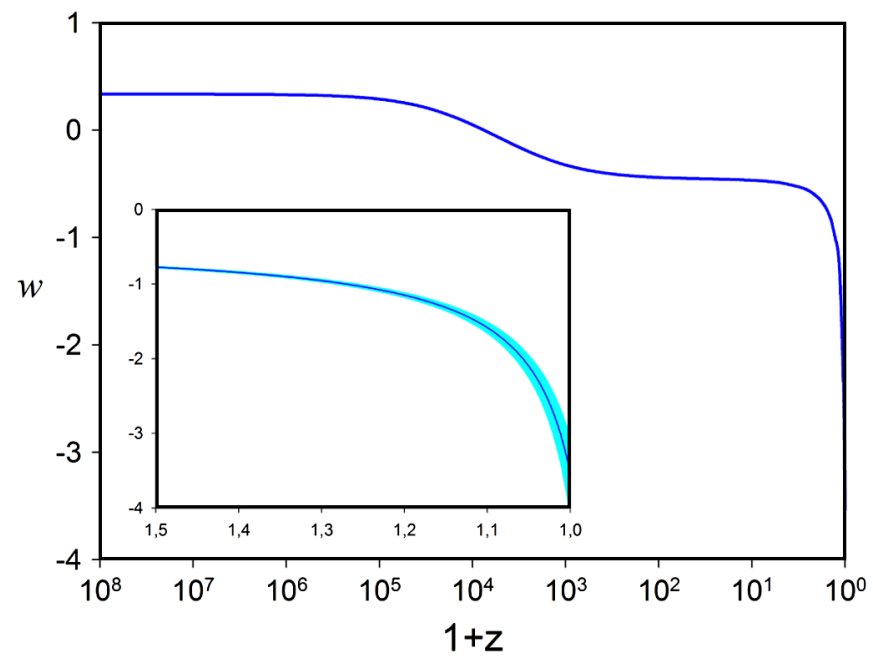

FIG. 2 (color online). Evolution of dark energy equation of state for the best-fit model. The lower panel shows the $1 \sigma$ confidence interval.

novae: the Gold set [14], containing 157 points with $z<$ 1.7, and the more recent Supernovae Legacy Survey (SNLS) data set [15], comprising 115 supernovae but with lower redshifts $(z<1)$.

In Table I we show the results for the best fit together with its corresponding $1 \sigma$ intervals for the two data sets. We also show for comparison the results for a standard $\Lambda \mathrm{CDM}$ model. We see that the vector model (VCDM) fits the data considerably better than $\Lambda \mathrm{CDM}$ (at more than $2 \sigma$ ) in the Gold set, whereas the situation is reversed in the SNLS set. This is just a reflection of the well-known $2 \sigma$ tension [16] between the two data sets. The best-fit parameters for the VCDM model are identical for the two data sets with small differences in the confidence intervals. Compared with $\Lambda \mathrm{CDM}$, we see that VCDM favors a younger universe (in $H_{0}^{-1}$ units) with larger matter density. In addition, the deceleration-acceleration transition takes

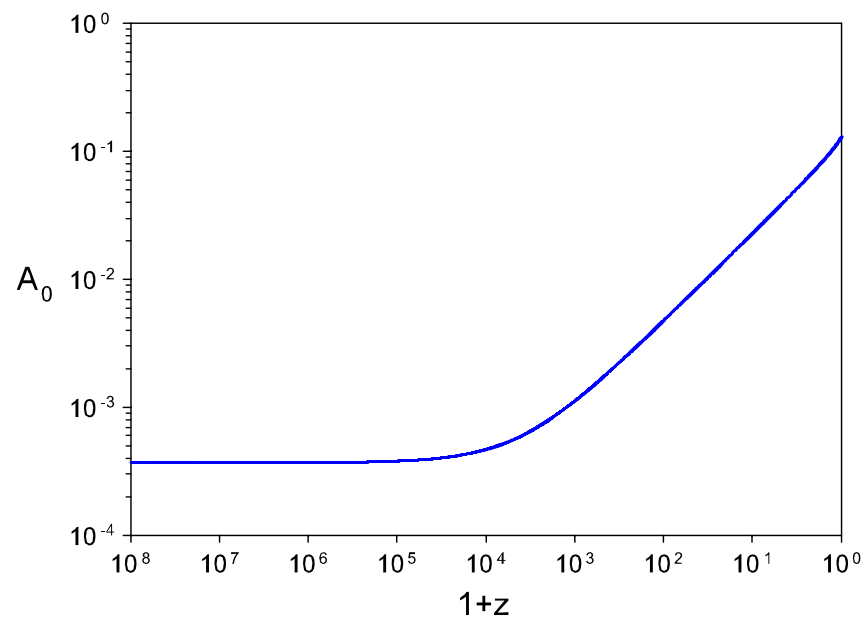

FIG. 3 (color online). Evolution of $A_{0}$ in $M_{P}$ units for the bestfit model. place at a lower redshift in the VCDM case. Another important difference arises in the present value of the equation of state with $w_{0}=-3.53_{-0.57}^{+0.46}$ which clearly excludes the cosmological constant value -1 . Future surveys [17] are expected to be able to measure $w_{0}$ at the few percent level and therefore could discriminate between the two models.

We have also compared with other parametrizations for the dark energy equation of state. Thus, for instance, taking $w_{\mathrm{DE}}(z)=w_{0}+w_{1} z(1+z)^{-1}$ [18], we find $\chi^{2}=173.5$ for the Gold set. Since this is a three-parameter fit, in order to compare with the one-parameter fits of VCDM or $\Lambda \mathrm{CDM}$, we use the reduced chi-squared: $\chi^{2} /$ d.o.f $=$ 1.108 for $\mathrm{VCDM}, \chi^{2} /$ d.o.f $=1.127$ for the $\left(w_{0}, w_{1}\right)$ parametrization, and $\chi^{2} /$ d.o.f $=1.135$ for $\Lambda$ CDM. As a matter of fact, to our knowledge best, VCDM provides the best fit to date for the Gold data set, since the oscillatory four-parameter model previously reported in [19] still has $\chi^{2} /$ d.o.f $=1.115$.

The evolution of dark energy for the best-fit model is plotted in Figs. 1-3. We see that unlike the cosmological constant case, throughout the radiation era $\rho_{\mathrm{DE}} / \rho_{R} \sim$ $10^{-6}$. Notice that although the onset of cosmic acceleration depends on the value of $A_{0}$ during that era, for the best-fit $A_{0}=3.71 \times 10^{-4} M_{P}$, which is relatively close to the Planck scale and could arise naturally in the early universe without the need of introducing extremely small parameters.

When comparing the parameters obtained from SN Ia (Table I) with predictions coming from cosmic microwave background (CMB) anisotropies or baryon acoustic oscillations [20], it is important to keep in mind that such predictions are obtained after a data process which involves the use of a particular model for dark energy, which in most cases is $\Lambda \mathrm{CDM}$, as a fiducial model. This is a good approximation for models with (nearly) constant equation of state [20], but could not be a priori justified in our case since $w_{\mathrm{DE}}(z)$ has a strong redshift dependence [21].

In this work we have only considered the time component of the vector field. The presence of spatial components could, in principle, have adverse effects. However, we have found that the energy density of the spatial part decays as $a^{-8}$ during radiation and matter eras, i.e. much faster than the temporal contribution, so that it will not dominate at late times. On the other hand, we have calculated the evolution of $\left(p_{\|}-p_{\perp}\right) / \rho$, where $p_{\|}$is the pressure along the direction of the spatial component and $p_{\perp}$ is the transverse pressure, and we have found that this quantity decays very fast during the matter and radiation eras. Accordingly, we do not expect the generation of large anisotropies.

So far we have only considered the homogeneous model. In order to study the model stability we have considered the evolution of metric and vector field perturbations. Thus, we obtain the dispersion relation and the propagation speed of 
TABLE I. Best-fit parameters with $1 \sigma$ intervals for the vector model (VCDM) and the cosmological constant model ( $\Lambda$ CDM) for the Gold $(157 \mathrm{SNe})$ and SNLS $(115 \mathrm{SNe})$ data sets. $w_{0}$ denotes the present equation of state of dark energy. $A_{0}$ is the constant value of the vector field component during radiation. $z_{T}$ is the deceleration-acceleration transition redshift. $t_{0}$ is the age of the Universe in units of the present Hubble time. $t_{\text {end }}$ is the duration of the Universe in the same units.

\begin{tabular}{|c|c|c|c|c|}
\hline & VCDM Gold & $\Lambda$ CDM Gold & VCDM SNLS & $\Lambda$ CDM SNLS \\
\hline$\Omega_{M}$ & $0.388_{-0.024}^{+0.023}$ & $0.309_{-0.037}^{+0.039}$ & $0.388_{-0.020}^{+0.022}$ & $0.263_{-0.036}^{+0.038}$ \\
\hline$w_{0}$ & $-3.53_{-0.57}^{+0.46}$ & -1 & $-3.53_{-0.48}^{+0.44}$ & -1 \\
\hline$A_{0}\left(10^{-4} M_{P}\right)$ & $3.71_{-0.026}^{+0.022}$ & $\cdots$ & $3.71_{-0.024}^{+0.020}$ & $\cdots$ \\
\hline$z_{T}$ & $0.265_{-0.012}^{+0.011}$ & $0.648_{-0.095}^{+0.101}$ & $0.265_{-0.012}^{+0.010}$ & $0.776_{-0.108}^{+0.120}$ \\
\hline$t_{0}\left(H_{0}^{-1}\right)$ & $0.926_{-0.023}^{+0.026}$ & $0.956_{-0.032}^{+0.035}$ & $0.926_{-0.022}^{+0.022}$ & $1.000_{-0.037}^{+0.041}$ \\
\hline$t_{\text {end }}\left(H_{0}^{-1}\right)$ & $0.976_{-0.014}^{+0.018}$ & $\cdots$ & $0.976_{-0.013}^{+0.015}$ & $\cdots$ \\
\hline$\chi_{\min }^{2}$ & 172.9 & 177.1 & 115.8 & 111.0 \\
\hline
\end{tabular}

scalar, vector, and tensor modes. For all of them we obtain $v=\left(1-16 \pi G A_{0}^{2}\right)^{-1 / 2}$ which is real throughout the Universe evolution, since the value $A_{0}^{2}=(16 \pi G)^{-1}$ exactly corresponds to that at the final singularity. Therefore the model does not exhibit exponential instabilities. As shown in [22], the fact that the propagation speed is faster than $c$ does not necessarily imply inconsistencies with causality. We have also considered the evolution of scalar perturbations in the vector field generated by scalar metric perturbations during the matter and radiation eras, and found that the energy density contrast $\delta \rho_{A} / \rho_{A}$ is constant on super-Hubble scales, whereas it oscillates with growing amplitude as $a^{2}$ in the radiation era and as $\sim a^{0.3}$ in the matter era for sub-Hubble scales. Therefore again, we do not find exponentially growing modes.

The model proposed in this work can be considered as an effective description of dark energy on cosmological scales. Extending the applicability range to smaller scales requires consistency with local gravity tests. Indeed, we can see that for the model in (1), the static post-Newtonian parameters agree with those of general relativity [12], i.e. $\gamma=\beta=1$. For the parameters associated to preferred frame effects we get: $\alpha_{1}=0$ and $\alpha_{2}=8 \pi A_{\odot}^{2} / M_{P}^{2}$ where $A_{\odot}^{2}$ is the norm of the vector field at the solar system scale. Current limits $\alpha_{2} \lesssim 10^{-4}$ (or $\alpha_{2} \lesssim 10^{-7}$ for static vector fields during solar system formation) then impose a bound $A_{\odot}^{2} \lesssim 10^{-5}\left(10^{-8}\right) M_{P}^{2}$, which could conflict with the model predictions, since the present (solar system formation) values on cosmological scales are: $1.3 \times 10^{-1} M_{P}(7.5 \times$ $\left.10^{-2} M_{P}\right)$. However, notice that the cosmological values do not need to agree with those at lower scales. The latter will be determined by the mechanism that generated this field in the early Universe characterized by its primordial spectrum of perturbations, and the subsequent evolution in the formation of the galaxy and solar system. Concerning the potential presence of quantum instabilities in the model, in [23] the condition in order to ensure positive norm Hilbert space is obtained. With our Riemann tensor sign convention, we see that such a condition is indeed satisfied in the model (1).

In conclusion, the results of this work show that vector theories offer an accurate phenomenological description of dark energy in which fine-tuning problems could be avoided.

This work has been supported by DGICYT (Spain) Project No. FPA 2004-02602 and No. FPA 2005-02327, UCM-Santander PR34/07-15875, and by CAM/UCM 910309. J. B. acknowledges support from MEC Grant No. BES-2006-12059.
[1] S. Perlmutter et al., Astrophys. J. 517, 565 (1999).

[2] A. G. Riess et al., Astron. J. 116, 1009 (1998); 117, 707 (1999).

[3] D. N. Spergel et al., Astrophys. J. Suppl. Ser. 148, 175 (2003); 170, 377 (2007).

[4] M. Tegmark et al., Phys. Rev. D 69, 103501 (2004).

[5] C. Wetterich, Nucl. Phys. B302, 668 (1988); R. R. Caldwell, R. Dave, and P. J. Steinhardt, Phys. Rev. Lett. 80, 1582 (1998).

[6] C. Armendariz-Picon, T. Damour, and V. Mukhanov, Phys. Lett. B 458, 209 (1999).
[7] S. M. Carroll, V. Duvvuri, M. Trodden, and M. S. Turner, Phys. Rev. D 70, 043528 (2004).

[8] G. Dvali, G. Gabadadze, and M. Porrati, Phys. Lett. B 485, 208 (2000).

[9] V. V. Kiselev, Classical Quantum Gravity 21, 3323 (2004).

[10] C. Armendariz-Picon, J. Cosmol. Astropart. Phys. 07 (2004) 007; C. G. Boehmer and T. Harko, Eur. Phys. J. C 50, 423 (2007); M. Novello et al., Phys. Rev. D 69, 127301 (2004); T. Koivisto and D.F. Mota, arXiv:0707.0279.

[11] P. G. Ferreira et al., Phys. Rev. D 75, 044014 (2007). 
[12] C. Will, Theory and Experiment in Gravitational Physics (Cambridge University Press, Cambridge, England, 1993).

[13] S. Nojiri, S. D. Odintsov, and S. Tsujikawa, Phys. Rev. D 71, 063004 (2005).

[14] A. G. Riess et al., Astrophys. J. 607, 665 (2004).

[15] P. Astier et al., Astron. Astrophys. 447, 31 (2006).

[16] S. Nesseris and L. Perivolaropoulos, J. Cosmol. Astropart. Phys. 02 (2007) 025.

[17] R. Trotta and R. Bower, Astron. Geophys. 47, 4.20 (2006).

[18] M. Chevalier and D. Polarski, Int. J. Mod. Phys. D 10, 213
(2001); E. V. Linder, Phys. Rev. Lett. 90, 091301 (2003).

[19] R. Lazkoz, S. Nesseris, and L. Perivolaropoulos, J. Cosmol. Astropart. Phys. 11 (2005) 010.

[20] D. J. Eisenstein et al., Astrophys. J. 633, 560 (2005).

[21] J. Dick, L. Knox, and M. Chu, J. Cosmol. Astropart. Phys. 07 (2006) 001.

[22] E. Babichev, V. Mukhanov, and A. Vikman, J. High Energy Phys. 02 (2008) 101.

[23] B. M. Gripaios, J. High Energy Phys. 10 (2004) 069. 